\title{
A treatment protocol for abdomino-pelvic injuries
}

\author{
Alberto Nicodemo · Daniela Decaroli · Jacopo Pallavicini · \\ Roberto Sivieri $\cdot$ Alessandro Aprato $\cdot$ Alessandro Massè
}

Received: 16 August 2006/Accepted: 2 April 2008/Published online: 14 May 2008

(C) Springer-Verlag 2008

\begin{abstract}
Background Abdomino-pelvic injuries often present a challenge for the emergency department. Although literature reports several protocols on the treatment of abdomino-pelvic injuries aiming at defining the most advisable treatment line, optimal treatment is still controversial. This paper describes a protocol that has been used to treat abdomino-pelvic injuries in our hospital since 2002. Materials and methods In literature different protocol of abdomino-pelvic injuries are described and comparing them most of the difference are the timing of CT scan, the angiography and the laparotomy when treating a lesion of pelvic ring. If patient is haemodynamically instable and
\end{abstract}

A. Nicodemo

Orthopaedic Department,

San Giovanni Bosco Hospital of Turin, Turin, Italy

D. Decaroli

Department of Emergency, Intensive Care Unit,

CTO Hospital, Turin, Italy

J. Pallavicini

Department of General Surgery, CTO Hospital, Turin, Italy

R. Sivieri

Metabolic Disease and Diabetology, CTO Hospital, Turin, Italy

A. Aprato

Faculty of Medicine and Surgery,

University of Turin, Turin, Italy

\section{A. Massè $(\square)$}

1st Orthopaedic Clinic (Director P. Gallinaro),

I Clinica Ortopedica e Traumatologica,

University of Turin, Via Zuretti 29, 10122 Torino, Italy

e-mail: alessandro.masse@unito.it presents a lesion of pelvic ring our protocol suggest the simplest and fastest stabilization (pelvic external fixator) in emergency room and delay exam such as CT scan as second level exam. In the presence of an abdominal injury, with a positive focused assessment with sonography for trauma test, the first step should be a pelvic ring stabilization, as laparotomy decreases the abdominal pressure and reduces the tamponade effect on the retroperitoneum. According to presented protocol the angiography is not be a first choice treatment. This protocol was applied to 58 cases of abdomino-pevic injury with unstable pelvic lesions from October 2002 to December 2005. Mean injury severity score was 27.2 (CI 24.1-30.3).

Results Five patients (8\%) died, three due to haemorrhagic shock and two due to pulmonary embolization. Four patients $(6.9 \%)$ had a partial or complete cauda equina syndrome, four patients (6.9\%) complained of mild incontinence, whilst 1 (1.7\%) complained of urinary retention with multiple cystitis. Two patients $(3.4 \%)$ with retention and multiple cystitis, had a malunion and a painful non-union of the fracture. Seven patients (12.3\%) had neurological impairment: $5(8.6 \%)$ sciatic nerve palsy, $1(1.7 \%)$ lumbosacral root lesions in a C2-type fracture and there was one case $(1.7 \%)$ of inconstant lumbago with sciatic pain. Twelve patients reported different levels of sexual dysfunction (20.7\%).

Conclusions Although validation with a larger cohort is required, our preliminary clinical data are similar to, or better than, those reported in the most recent publications on this question, suggesting that this protocol could well reduce both the mortality rate and the long term complications of abdominopelvic injuries.

Keywords Algorithm - Fracture - Injury · Pelvis · Protocol 


\section{Introduction}

As abdomino-pelvic injuries must be cared for by a multidisciplinary team, made up of an orthopaedic trauma surgeon, a general surgeon, an anaesthesiologist, a radiologist and an angiographist (and, at times, also a urologist and a gynaecologist), this pathology often presents a challenge for the emergency department [4].

Although the presence of all these specialists allows for complete treatment, it may also create confusion and lead to the deferring of proper decisions $[5,33]$. Such delays are, however, not acceptable with these injuries, as patients are frequently in critical conditions and require a rapid, correct diagnosis and therapy. Statistics have placed these injuries in the third place as cause of death in motorcycle accidents and the mortality rate of unstable pelvic ring fractures is as high as $20 \%$ [10]. That is why many authors $[3,6,7,14,27,37]$ are of the opinion that this kind of trauma should be immediately referred to specialized centres where diagnostic protocols and treatment are used.

All the protocols $[3,6,7,14,27,37]$ reported in literature are based on the "damage control orthopaedics (DCO)" [18, 34]: this means that any intervention should be rapid and minimally traumatic focusing on haemorrhage control and on other life saving measures. The surgical burden on the immune response and that caused by the primary injury, are considered critical factors that have a direct effect on the patient's clinical course. Sub-clinical consequences of the initial trauma and/or those due to surgery may manifest themselves as abnormalities in organ function, leading to Multiple Organ Dysfunction syndrome (MODS). That is why definitive surgical treatment should be kept to a minimum in multiply injured patients i.e. complex reconstructive operations should be postponed until such times as the patient is haemodynamically stable and in a better general condition.

There are several treatment options that should be considered for the emergency haemostasis of pelvic fractures, such as: the pelvic sling, arterial inflow arrest, external fixation devices, internal fixation, direct surgical haemostasis, pelvic packing, pelvic angiography and embolization. Only external devices that can be easily applied can be used effectively [11, 12, 17, 21, 24, 28, 31, 37]. These devices create a tamponade effect against ongoing bleeding by reducing the intrapelvic volume and also restore stability, bone contact and favour blood clotting. Should the bleeding continue despite the application of the external fixator, then pelvic packing should be considered, as should tamponade of the areas, or temporary aortic compression, in the presence of multiple massive bleeding points. Complex abdominal procedures should be avoided in the presence of pelvic haemorrhage $[2,9]$. A major spleen rupture usually necessitates splenectomy. In liver injuries, attention is paid only to major vessels and hepatic tamponade is applied [19]. Bowel injuries are clamped and covered, leaving definitive treatment until such times as the haemodynamic situation has been stabilized. As angiographic embolization is both time consuming and inhibitive to dynamic assessment and further treatment, it is not usually indicated in this population. However, in cases where haemodynamic stability with volume replacement can be achieved, but ongoing pelvic haemorrhage is suspected (expanding haematoma), it is a good practise to add angiography to the treatment protocol [9]. The management of the pelvic fracture should be conceived as part of the resuscitative effort. Immediate external fixation of the unstable pelvis with pelvic packing to control pelvic haemorrhage is a practical approach in both the borderline and "in extremis" patient. Angiographic embolization can be recommended in more stable patients. Any benefits of immediate fracture stabilization are obtained at an early stage i.e. a reduction in blood loss and, therefore the need for transfusion/s and/or a reduced risk of developing systemic complications.

The guidelines reported in literature have advocated the principles shown in Table 1 [2, 9, 19]. Therefore, in an effort to optimise the emergency department resources, reduce the mortality and morbidity rate of this trauma (and, consequentially the high costs to society), a team of orthopaedic surgeons, anaesthesiologists and general surgeons applied these principles to form the basis of a treatment protocol for abdomino-pelvic injuries. This Protocol has been routinely applied at the Orthopaedic and Traumatologic Hospital of Turin (CTO Hospital) since October 2002 to time of writing.

\section{Materials and methods}

A review of international literature on abdomino-pelvic injuries and pelvic ring fractures was carried out through Pub Med with the following medical subject heading (MeSH): blunt abdominal trauma, pelvic fracture, pelvic trauma, pelvic injury, pelvic ring disruption terms. Case reports and small series revisions were excluded and only articles in the English language, or published after 1995 were included. The resulting data formed the basis of our protocol.

\section{Clinical management}

First step is the ABCDE (airway, breathing, circulation, disability, and exposure/environment control) assessment, according to the advanced trauma life support (ATLS) guidelines [9] and includes a haemodynamic stability evaluation. In the presence of any one, or a combination of, the following criteria a patient is considered haemodynamically unstable: a systolic blood pressure below $90 \mathrm{mmHg}$, a heart beat rate above $110 \mathrm{bpm}$, or in the presence of clinical signs of insufficient organ perfusion 
Table 1 The guideline principles with a high level of evidence [25, 26, 27, 37]

CT is recommended for the evaluation of haemodynamically stable patients with equivocal findings on physical examination,
associated neurological injury, or multiple extra-abdominal injuries. Under these circumstances, patients with a negative
CT should be admitted for observation. In haemodynamically stable patients the CT is a complementary diagnostic modality
Exploratory laparotomy is indicated in haemodynamically unstable patients with a positive FAST. In haemodynamically stable
patients with a positive FAST, follow-up CT allows for a non-operative management of select injuries [9]
A negative FAST should prompt follow-up CT for patients at high risk for intra-abdominal injuries (e.g., multiple orthopaedic
injuries, severe chest wall trauma, and neurological impairment)
Patients with a major pelvic fracture with signs of on going bleeding after non-pelvic sources of blood loss have been ruled out
should be considered for pelvic angiography and possible embolization
4 Patients with evidence of unstable fractures of the pelvis associated with hypotension should be considered for some form of
external pelvic stabilization
Patients with evidence of unstable pelvic fractures who warrant laparotomy should receive external pelvic stabilization prior
to laparotomy incision
Patients with major pelvic fracture who are found to have bleeding in the pelvis, which cannot be adequately controlled
at laparotomy, should be considered for pelvic angiography and possible embolization
Patients with evidence of arterial extravasation of intravenous contrast in the pelvis by computed tomography should
be considered for pelvic angiography and possible embolization
Patients with hypotension and gross blood in the abdomen or evidence of intestinal perforation warrant emergent laparotomy
Urgent laparotomy is warranted for patients who demonstrate signs of continued intra-abdominal bleeding after adequate
resuscitation, or evidence of intestinal perforation

(oliguria, small vessel vasoconstriction, or mental confusion not due to intoxication or head trauma) [8, 13, 15, 25] even after ATLS resuscitation and infusions.

The "in extremis" patient with massive blood loss and hypovolaemic shock is immediately taken to the operating theatre for a "blitz laparotomy" (i.e. clamping the aorta below the renal vessels).

The haemodynamically unstable patient

In the case of a haemodynamically unstable patient who is not in shock, only the easiest and fastest exams are performed, without moving the patient from the emergency room (ER) table i.e. an antero-posterior (AP) pelvis X-ray and a focused assessment with sonography for trauma (FAST) test [26]. Depending on the results of these examinations, four scenarios may be defined.

1. Both X-ray and FAST are negative: in this case other sources of bleeding have to be considered and the FAST is repeated within $1 \mathrm{~h}$.

2. The X-ray is positive for an unstable pelvic fracture (Tile B or C) and FAST is negative: in this case the pelvis is quickly stabilized by an external fixator or pelvic clamp, before any other manoeuvre is made.

3. The X-ray is negative but the FAST test is positive: this patient is taken to the operating theatre for a laparotomy, which is performed according to the principles of the damage control surgery.

4. Both the X-ray and FAST are positive: in this scenario, again, the pelvis is quickly stabilized by an external fixator or pelvic clamp: the easiest stabilization, which can be performed directly in the emergency department without fluoroscopy, is to insert two pins into the iliac crest. If the patient is still haemodynamically unstable after pelvic stabilization, or if the FAST is highly suspicious for an abdominal organ rupture, a laparotomy is carried out. This sequence is possible only if a well trained orthopaedic surgeon performs the pelvic stabilization within a few minutes, otherwise a laparotomy, pelvic packing and treatment of any organ injuries must be done first.

Haemodynamic stability must be re-assessed after each step.

Should the patient still be unstable, but other sources of bleeding can be excluded, then an angiography must be done. It is advisable to repeat the FAST test in cases 1 and 2, as some parenchymal lesions do not bleed heavily immediately and may give a first negative ultrasonography (US).

The haemodynamically stable patient

If the patient is haemodynamically stable, then a little more time may be spent on other examinations. It is, therefore, essential to monitor the vital signs continuously. Also in this case the first exams are an AP pelvis X-ray and a FAST test. If the US is positive, then it is necessary to ask for a CT of the pelvis and abdomen, as the CT has a higher specificity than FAST and can offer more information as to the pelvic fracture. A laparotomy and/or a pelvic ring stabilization may be done, depending on the results of the CT. Should both be required, then the bone takes priority due to the reasons explained before. 
A definitive stabilization (internal fixation with sacroiliac joint screws, symphysis plate etc.) [20, 22, 23, 32, 35, 36] may be performed if: (1) a surgeon well versed in pelvic fracture is available (2) the general conditions of the patient are not critical (3) there is a negative US and a B or $\mathrm{C}$ type fracture. The proposed algorithm is summarized in Fig. 1. Figure 2 shows an example case.

\section{Patients}

A clinical retrospective study was set up to support the proposed protocol. Fifty-eight cases (37 males/21 females) of abdomino-pelvic injury with unstable pelvic lesions were treated, according to the aforementioned described protocol, from October 2002 to December 2005. Most percentage of the cases was motorcycle accidents (43\%), followed by car accidents $(27 \%)$, falls from a significant height $(16 \%)$ and other causes $(14 \%)$. According to the Tile's classification, there were 37 type B lesions (horizontal instability) and 21 type $\mathrm{C}$ (global instability). The average age was 43 years (18-76) and the average followup was 15.5 months (range 7-38). Thirty-five $(60 \%)$ patients were haemodynamically stable and $55(95 \%)$ required blood transfusion. There were 26 associated

Fig. 1 Treatment protocol

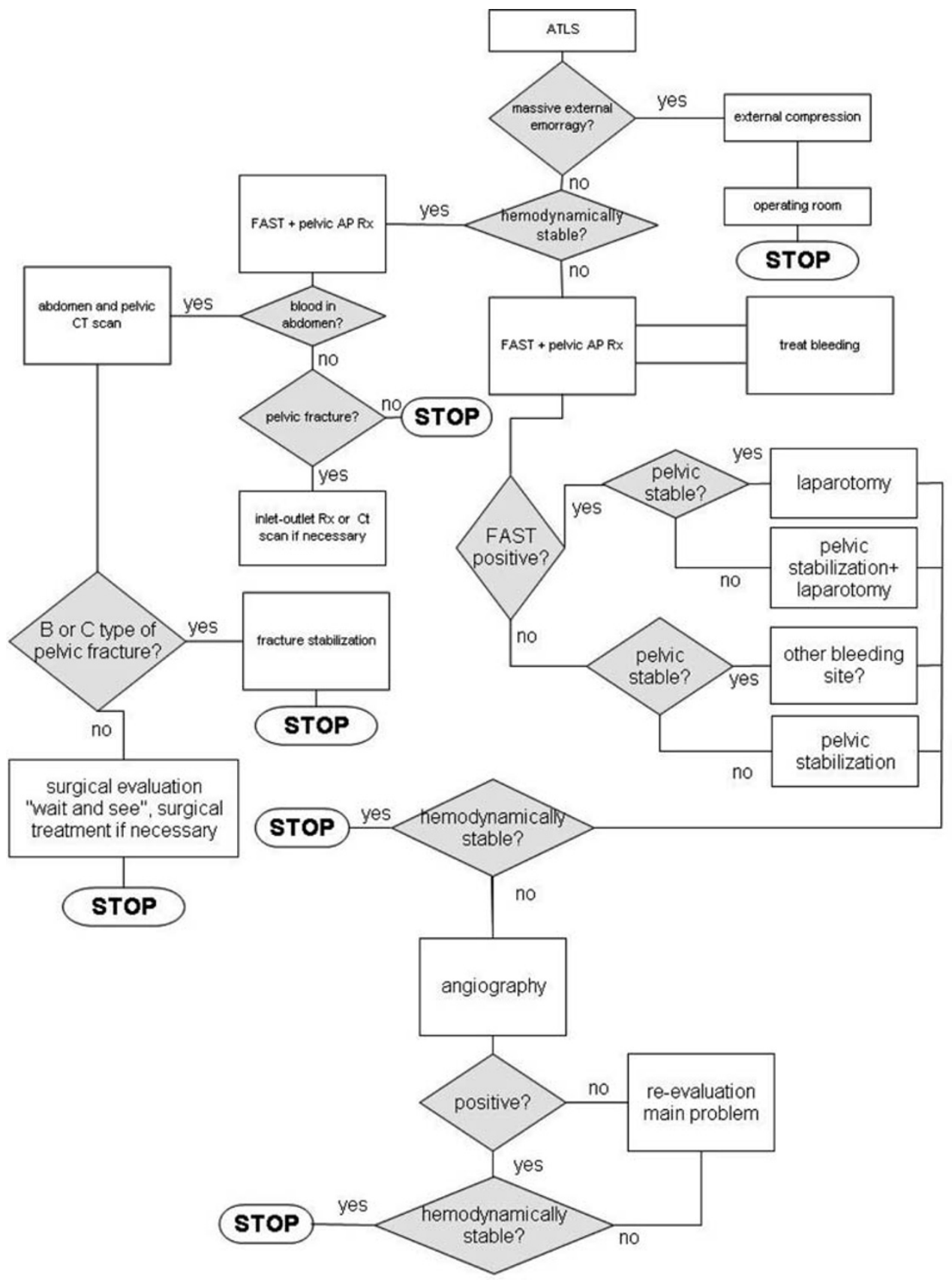


Fig. 2 A 35-year-old man, involved in car accident, reported an unstable pelvic fracture (tile C1.1), femoral fracture, proximal humeral fracture and a mesenterial lesion. Pelvic fixation was performed a X-ray in anteroposterior view, $\mathbf{b}$ in outlet view and $\mathbf{c}$ in inlet before laparotomy d
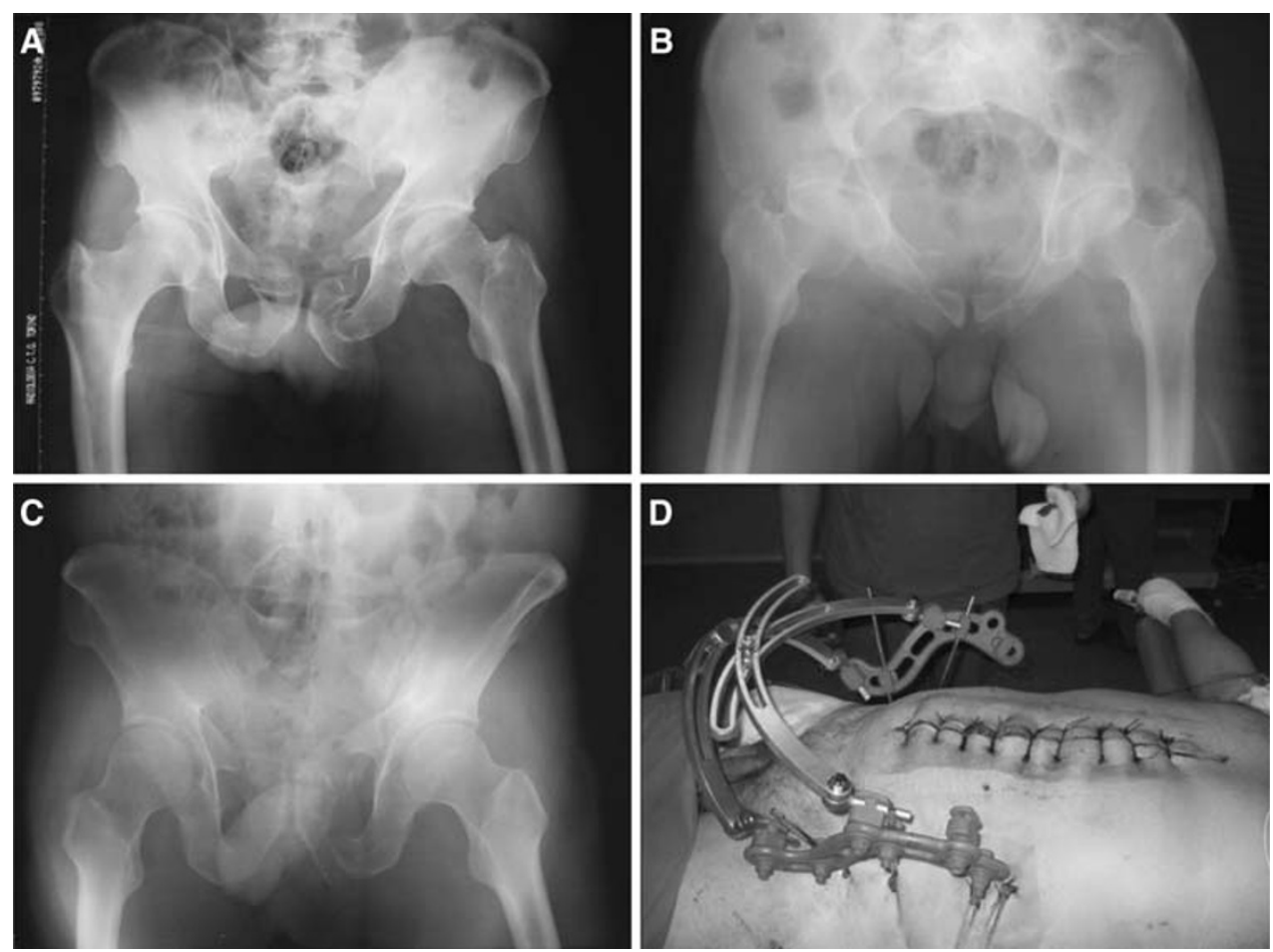

lesions: six spleen ruptures, three liver ruptures, three diaphragm lacerations with liver herniation, three bladder ruptures, four urethral partial lesions, two urethral disjunctions and five cases of severe brain injury.

Mean injury severity score (ISS) was 27.2 (CI 24.130.3). All the pelvic lesions were treated by external fixation; laparotomy was performed in 14 cases and angiographic embolization in four.

All the patients included in the study gave their informed consent. The study was authorized by the local ethical committee and was performed in accordance with the Ethical standards of the 1964 Declaration of Helsinki.

\section{Results}

There was an $8.6 \%$ mortality rate: five patients died, three due to haemorrhagic shock and two due to pulmonary embolization.

Four patients $(6.9 \%)$ had a partial or complete cauda equina syndrome, due to multiple damage of the sacral roots due to sacral fracture. Four patients $(6.9 \%)$ complained of mild incontinence, whilst $1(1.7 \%)$ complained of urinary retention with multiple cystitis.

Two patients $(3.4 \%)$ with retention and multiple cystitis, had a malunion and a painful non-union of the fracture. Correction of the deformities (osteotomy and internal fixation) partially resolved the urinary problems.
Seven patients $(12.3 \%)$ had neurological impairment: 5 $(8.6 \%)$ sciatic nerve palsy, 1 (1.7\%) lumbosacral root lesions in a C2-type fracture and there was one case $(1.7 \%)$ of inconstant lumbago with sciatic pain.

Twelve patients reported different levels of sexual dysfunction $(20.7 \%)$ : in this cohort three female patients complained of dispareunia due to a mal-united fracture of the ileopubic branch. Two wound infections $(3.4 \%)$ of the Pfannestiel approach were recorded and were resolved by topical medication.

All haemodynamically unstable patients had surgical stabilization of the pelvis within two hours from the arrival in the emergency department. Thirty-six (62\%) of the lesions (15 type $\mathrm{B}$ and all the type $\mathrm{C}$ fractures) underwent further surgery at an average of 4 days from the first stabilization.

\section{Discussion}

Several protocols on the treatment of abdomino-pelvic injuries have been published in an effort to define the best sequence of action, depending on the different scenarios. However, these protocols are of difficult implementation in clinical practice as the resources available to deal with this pathology differ from hospital to hospital.

The clinical protocol herein reported was established on the evidence-based knowledge in abdomino-pelvic injury 
Table 2 A comparison of the protocols presented in literature

\begin{tabular}{|c|c|c|c|c|c|}
\hline Reference & Angiography & Laparotomy & $\begin{array}{l}\text { Percentage } \\
\text { mortality }\end{array}$ & C.T. scan/FAST/Dpl & $\begin{array}{l}\text { External fixation/pelvic } \\
\text { clamp }\end{array}$ \\
\hline 1 & FAST negative & FAST positive & 15 & $\begin{array}{l}\text { FAST always, CT scan if } \\
\text { haemodynamically stable }\end{array}$ & $\begin{array}{l}\text { Depending on fracture } \\
\text { pathway }\end{array}$ \\
\hline 8 & All unstable patients & Not specified & 11 & $\begin{array}{l}\text { DPL if haemodynamically unstable, } \\
\text { CT scan if stable }\end{array}$ & Urgent ORIF \\
\hline 10 & Last step & FAST positive & n.s. & NS & Unstable fracture \\
\hline 14 & DPL negative & DPL positive & $32-45$ & $\begin{array}{l}\text { DPL if haemodynamically unstable, } \\
\text { CT scan if stable }\end{array}$ & Unstable fracture \\
\hline 37 & DPL negative & DPL negative & 47 & $\begin{array}{l}\text { CT scan if haemodynamically stable, } \\
\text { DPL if unstable }\end{array}$ & Not used \\
\hline 38 & DPL negative & DPL negative & n.s. & $\begin{array}{l}\text { DPL or FAST if haemodynamically } \\
\text { unstable, CT scan if stable }\end{array}$ & First step \\
\hline OUR & Last step & FAST positive & 8.6 & $\begin{array}{l}\text { FAST always, CT scan if } \\
\text { haemodynamically stable }\end{array}$ & First step \\
\hline
\end{tabular}

FAST DPL diagnostic peritoneal lavage, ORIF open reduction and internal fixation, $N S$ not specified

so as to take advantage of the best resources available in our hospital by co-ordinating them in the emergency department. We are of the opinion that this protocol is easily adaptable to most trauma centres, as the first steps are based on standard diagnostic examinations and therapeutic procedures. Indeed, more complex procedures, such as angiography, are required only in the very last steps.

Table 2 shows a comparison between the most commonly used protocols.

Indications as to when an external fixator, or a pelvic clamp should be used, why and when an angiography or a laparotomy should be done, are compared shown in Table 2 [2, 9, 11, 12, 17, 19, 21, 24, 26, 28-31, 35-37]. Other factors considered in the comparison are the use of CT scan, or FAST and mortality rates.

The mortality rate, obtained in our study, compared favourably with those reported in literature, and showed a decrease of mortality respect other papers even if correlated with injury severity score. In fact most of reported case [1, 8, 14] showed an ISS of about 29 points on average, just slightly higher then 27 points obtained in this study but included in the calculated confidence interval.

Higher mortality rate was reported by Agolini [1] but his paper evaluated patients with a mean ISS of about 38 points; while other authors did not indicate the ISS [16, 37].

Although a validation with a larger cohort in a prospective study is advisable, these preliminary clinical data are encouraging, as they are similar to, or better than, those reported in updated literature, suggesting that this protocol may be effective in reducing the mortality rate.

Conflict of interest statement The authors declare that they have no conflict of interest related to the publication of this manuscript.

\section{References}

1. Agolini SF, Shah K, Jaffe J, Newcomb J, Rhodes M, Reed JF (1997) Arterial embolization is a rapid and effective technique for controlling pelvic fracture hemorrhage. J Trauma 43:395399

2. Alonso M, Brathwaite C, Garcia V, Patterson L, Scherer T, Stafford P, Young J. East Practice Management Guidelines Work Group: practice management guidelines for the nonoperative management of blunt injury to the liver and spleen

3. Bassam D, Cephas GA, Ferguson KA, Beard LN, Young JS (1998) A protocol for the initial management of unstable pelvic fractures. Am Surg 64:862-867

4. Biffl WL, Smith WR, Moore EE, Gonzalez RJ, Morgan SJ, Hennessey T, Offner PJ, Ray CE Jr, Franciose RJ, Burch JM (2001) Evolution of a multidisciplinary clinical pathway for the management of unstable patients with pelvic fractures. Ann Surg 233(6):843-850

5. Bircher M, Giannoudis PV (2004) Pelvic trauma management within the UK: a reflection of a failing trauma service. Injury $35(1): 2-6$

6. Burgess AR, Eastridge BJ, Young JW, Ellison TS, Ellison PS Jr, Poka A, Bathon GH, Brumback RJ (1990) Pelvic ring disruptions: effective classification system and treatment protocols. J Trauma 30(7):848-856

7. Cryer HM, Miller FB, Evers EM, Rouben LR, Seligson DL (1988) Pelvic fracture classification: correlation with hemorrhage. J Trauma 28:973-980

8. DiGiacomo JC, Bonadies JA, Cole FJ, Diebel L, Hoff WS, Holevar M, Malcynski J, Scalea T. East Practice Management Guidelines Work Group: practice management guidelines for hemorrhage in pelvic fracture

9. Gansslen A, Pohlemann T, Paul C, Lobenhoffer P, Tscherne H (1996) Epidemiology of pelvic ring injuries. Injury 27(Suppl 1):S-A13-S-A20

10. Ganz R, Krushell RJ, Jakob RP, Kuffer J (1991) The antishock pelvic clamp. Clin Orthop (267):71-78

11. Ghanayem AJ, Wilber JH, Lieberman JM, Motta AO (1995) The effect of laparotomy and external fixator stabilization on pelvic volume in an unstable pelvic injury. J Trauma 38:396-401

12. Grimm MR, Vrahas MS, Thomas KA (1998) Pressure-volume characteristics of the intact and disrupted pelvic retroperitoneum. J Trauma 44:454-459 
13. Gruen GS, Leit ME, Gruen RJ, Peitzman AB (1994) The acute management of hemodynamically unstable multiple trauma patients with pelvic ring fractures. J Trauma 36:706-713

14. Hamill J, Holden A, Paice R, Civil I (2000) Pelvic fracture pattern predicts pelvic arterial haemorrhage. Aust N Z J Surg 70(5):338-343

15. Heetveld MJ, Harris I, Schlaphoff G, Sugrue M (2004) Guidelines for the management of haemodynamically unstable pelvic fracture patients. ANZ J Surg 74(7):520-529

16. Heini PF, Witt J, Ganz R (1996) The pelvic C-clamp for the emergency treatment of unstable pelvic ring injuries. A report on clinical experience of 30 cases. Injury 27(Suppl 1):S-A38-S-A45

17. Hildebrand F, Giannoudis P, Kretteck C, Pape HC (2004) Damage control: extremities. Injury 35(7):678-689

18. Hoff WS, Holevar M, Nagy KK, Patterson L, Young JS, Arrillaga A, Najarian MP, Valenziano CP. East Practice Management Guidelines Work Group: practice management guidelines for the evaluation of blunt abdominal trauma

19. Kabak S, Halici M, Tuncel M, Avsarogullari L, Baktir A, Basturk M (2003) Functional outcome of open reduction and internal fixation for completely unstable pelvic ring fractures (type C): a report of 40 cases. J Orthop Trauma 17(8):555-562

20. Latenser BA, Gentilello LM, Tarver AA, Thalgott JS, Batdorf JW (1991) Improved outcome with early fixation of skeletally unstable pelvic fractures. J Trauma 31(1):28-31

21. Matta JM (1996) Indications for anterior fixation of pelvic fractures. Clin Orthop (329):88-96

22. Matta JM, Tornetta P (1996) 3rd Internal fixation of unstable pelvic ring injuries. Clin Orthop (329):129-140

23. Mears DC, Fu FH (1980) Modern concepts of external skeletal fixation of the pelvis. Clin Orthop (151):65-72

24. Moss MC, Bircher MD (1996) Volume changes within the true pelvis during disruption of the pelvic ring-where does the haemorrhage go? Injury 27(Suppl 1):S-A21-S-A23
25. Myers J (2007) Focused assessment with sonography for trauma (FAST): the truth about ultrasound in blunt trauma. J Trauma 62(6 Suppl):S28

26. Nerlich M, Maghsudi M (1996) Algorithms for early management of pelvic fractures. Injury 27(Suppl 1):S-A29-S-A37

27. Palmer S, Fairbank AC, Bircher M (1997) Surgical complications and implications of external fixation of pelvic fractures. Injury 28(9-10):649-653

28. Poole GV, Ward EF (1994) Causes of mortality in patients with pelvic fractures. Orthopedics 17:691-696

29. Riemer BL, Butterfield SL, Diamond DL, Young JC, Raves JJ, Cottington E, Kislan K (1993) Acute mortality associated with injuries to the pelvic ring: the role of early patient mobilization and external fixation. J Trauma 35(5):671-675 discussion 676677

30. Rubel IF, Kloen P, Borens O, Helfet DL (2002) External fixation for pelvic ring injuries. Tech Orthop 17(2):221-227

31. Shuler TE, Boone DC, Gruen GS, Peitzman AB (1995) Percutaneous iliosacral screw fixation: early treatment for unstable posterior pelvic ring disruptions. J Trauma 38(3):453-458

32. Solan MC, Molloy S, Packham I, Ward DA, Bircher MD (2004) Pelvic and acetabular fractures in the United Kingdom: a continued public health emergency. Injury 35(1):16-22

33. Sugrue M, D'Amours SK, Joshipura M (2004) Damage control surgery and the abdomen. Injury 35(7):642-648

34. Tile M (1988) Pelvic ring fractures: should they be fixed? J Bone Joint Surg Br 70(1):1-12

35. Tile M (1996) Acute pelvic fractures: II. Principles of management. J AAOS 4(3):152-161

36. Vrahas MS, Wilson SC, Cummings PD, Paul EM (1998) Comparison of fixation methods for preventing pelvic ring expansion. Orthopedics 21:285-289

37. Ward DA, Bircher MD (1996) The early management of pelvic and acetabular fractures. Injury 27(Suppl 1):S-A24-S-A28 